\title{
Evaluation of Interaction Methods for a Real-Time Augmented Reality Game
}

\author{
Frederic Pollmann, Dirk Wenig, Mareike Picklum, and Rainer Malaka \\ University of Bremen \\ Bibliothekstr. 1 \\ 28359 Bremen, Germany
}

\begin{abstract}
Augmented reality is a way to enhance mobile games and can be easily implemented on today's powerful smartphones. Developers need to consider additional constraints when choosing the input method for such an AR game. We implemented three control methods for a mobile AR multiplayer fighting game using a virtual joystick, a touch interface and continuous crosshair tracking. We evaluated the effect of the control methods on the game experience with 43 participants and conducted a survey using a questionnaire for intuitive use (QUESI [1]) and individiual interviews. We found significant differences between two of the three implemented input methods, but in the interviews the test persons did not prefer the control method with the highest survey score.
\end{abstract}

\section{Introduction}

Mobile devices such as smartphones are no longer just used for communications but also as a calendar, for browsing, email and entertainment. Their incorporated sensoring devices (eg. camera, GPS, microphone and acceleration sensor) and now powerful CPUs and large available memory enable them to run augmented reality $(\mathrm{AR})$ applications such as games. Overlaying the real world with an image the game world can provide a higher level of immersion in the game and make for a more enjoyable game experience. While it is possible to just use sensors such as an accelerometer or a compass for the synchronization of the real and the game world this approach does not yet yield compelling results, so a common solution is to use special markers that need to be tracked while running the AR application. We implemented and evaluated three different interaction methods to control the movement of an avatar in a mobile AR game. In it two players fight each other with an avatar in a fighting ring that is displayed in an AR environment. Attacking is controlled by a virtual button drawn on the screen. Our focus was to find an input method that let the players quickly learn the game and to concentrate on winning, not moving the avatar.

\section{Related Work}

Vaijk et al. 2] used the sensor data of a mobile phone to turn it into a motion controller similar to Nintendo's Wiimote. It was tested in a multiplayer racing 
game where it proved to be intuitively applicable as well as fun to use. Chehimi et al. 3] used the sensors of mobile phones for input in 3D multiplayer games. The users controlled a space ship in a space battle by moving the mobile phone. The authors concluded that 3D accelerometers provide new interaction possibilities and that motion controlled games can be fun to play. Duh et al. 4] studied the effect of innovative control methods on mobile devices on game experience. Games that require high eye-hand-coordination may be hard to play due to small screens, small keypads or control difficulties. They concluded that it may be better to stick to well-tried interaction concepts. Oda et al. [5] extended a car racing game by adding an augmented reality component. The car can be driven around on a tracked surface and is controlled using a handlebar with an additional marker that is also tracked. Hürst and van Wezel [6] used the camera to track the user's finger and use it to interact with the virtual world and control an avatar. A challenge using this interaction method was the difficulty to keep the device steady when moving the finger. Lagerstam et al. 7] tested camerabased interaction techniques with an AR application for children aged 10-11. Their results suggested that the mental overhead of both keeping a marker in the viewfield of a camera and trying to control some avatar in the AR world may be to high for children this age. Gu et al. [8] developed an AR game in which two players can fight each other in a virtual arena. To enhance game interactivity the accelerometer can be used to control the character. Multi-touch gestures could be used to control the character or initiate attacks.

When using a marker-based AR environment, the marker has to be visible to the devices camera at all times or the syncronization of virtual and real world is lost. This poses additional constraints on the player and the way he may play the game. Picklum et al. 9] proposed six different interaction methods to such a scenario. After considering the feedback to a poster presentation at ICEC 2012, three of the six methods were chosen and are evaluated in this paper.

\section{Interaction Methods}

For the evaluation of the input methods an existing mobile real-time augmented reality game 1 is used. The first control implemented is a touch-based virtual joystick that behaves similar to its hardware pendant found in common arcade or PC games. A circle is drawn on the touchscreen that symbolizes the movement range of the virtual joystick. The position of a player's finger inside the circle relative to its center defines the movement direction and speed of the avatar.

For the second control method the player sets the target of the avatar by touching the screen. The avatar moves towards the corresponding position in the AR world until it reaches it. Tapping on another point before the movement is completed interrupts this movement and sets a new target position for the avatar. This method will be referenced as "touchplane" in the rest of this paper. An example using this control method can be seen in Fig. 1,

\footnotetext{
1 The game is implemented using the graphics library libGDX and the AR framework Vuforia, both available for the mobile platform Android.
} 
The third control method uses the field of view of the mobile device's camera. A crosshair is drawn in the center of the display. The avatar continuously follows the point where this crosshair is projected to in the AR environment. Controlling the movement of the the avatar is achieved by moving or tilting the mobile device (and with it the integrated camera) while tracking the AR marker to move the crosshair to a new point in the AR world.

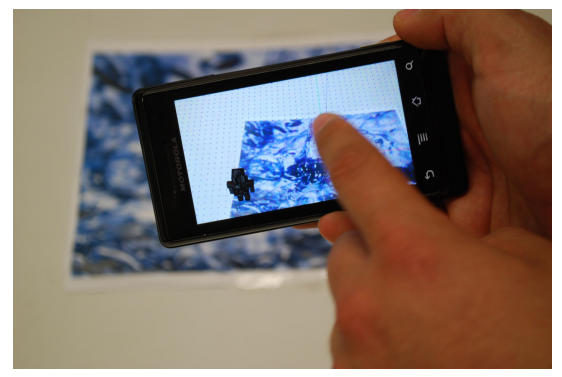

Fig. 1. The view of the game

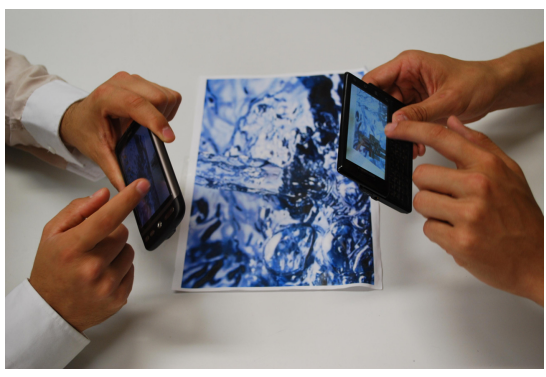

Fig. 2. The two player scenario

\section{Evaluation}

The evaluation was conducted by an observation of the test persons and a subsequent opinion survey about their personal attitude towards the control method that they used. A total number of 43 players took part in the experiment. 14 participants were female, 29 were male. The average age was 25 years (ranging from 17 to 49 years, $\sigma=5.5$ ). More than half (28) of the test subjects were students, the remaining subjects answered employed or miscellaneous as occupation. Participants came both from technical (eg. computer science or engineering) and non-technical fields (linguistics, geography or sports). Most (28) of the test persons regularly used a smartphone, but only nine of those regularly played games on it. 16 subjects played console games regularly, five for less than two hours, ten for two to ten hours and two for more than ten hours a week.

We divided the test subjects into two groups for a singleplayer (23 members) and a multiplayer (20 members) condition. In the first group the players had to defeat a simple computer-controlled enemy while in the second group two player were fighting each other as shown in Fig. 2. To remove the effect of the tension of a multiplayer battle from the evaluation, we only let players rate the intuitive usability after the singleplayer scenario using the Questionnaire for the Subjective Consequences of Intuitive use (QUESI) [1]. To make sure that the order of the presentation of the prototypes did not affect the result of the evaluation, we balanced the experiment using a latin squares [10] order. The participants of the multiplayer scenario were interviewed about their experience and asked for difficulties and possible improvements of the used input method. Additionally each test person was asked to give a personal ranking of the control methods at the end of each test. 


\section{Results and Analysis}

\subsection{Statistical Evaluation of the Singleplayer Condition}

In the singleplayer condition the joystick was rated as intuitively usable with a QUESI score of $3.81(\sigma=0.79)$, the touchplane with a score of $4.02(\sigma=0.73)$ and the crosshair with a score of $3.37(\sigma=0.90)$ (repeated-measures ANOVA, $F_{2,44}=4.670, p=0.014^{*}$. A detailed view of the scales that make up the QUESI score are shown in Fig. 3. When compared pairwise, the crosshair input method is significantly worse than the touchplane method $\left(p=0.009^{*}\right.$, Sidakcorrected). Comparing the different scale values of the QUESI results shows that the touchplane is significantly better than the joystick on every scale except for the perceived effort of learning and the perceived error rate $\left(p_{K}=0.017^{*}, p_{Z}=\right.$ $0.046^{*}, p_{L}=0.206, p_{V}=0.000^{*}, p_{F e}=0.110$ ). This suggests that the crosshair input method may be a new experience for the players that is easy to learn but not easy to master.

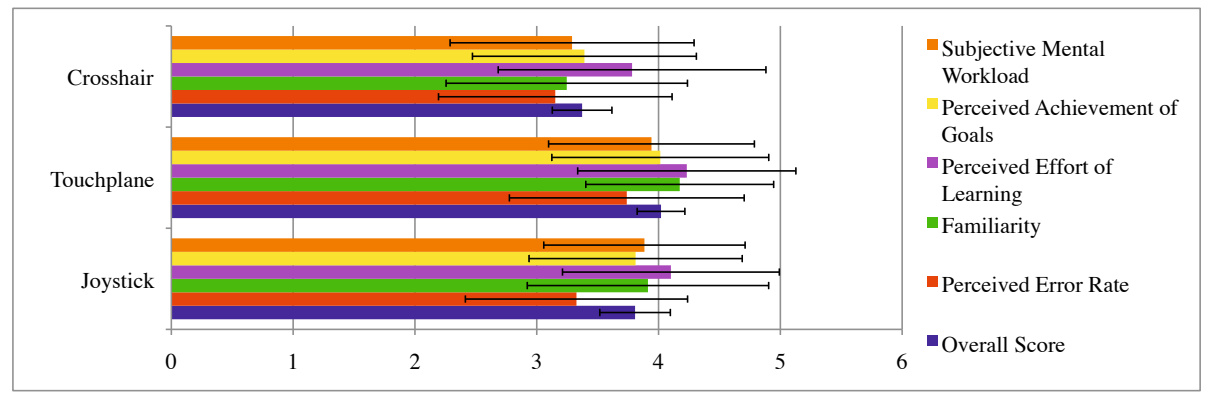

Fig. 3. Results of the QUESI survey of the input methods

\subsection{Interviews of the Participants of the Multiplayer Condition}

The interviews were conducted to get qualitative feedback on the different input methods.

Crosshair Interaction. Twelve participants thought of this control method as unusual and complicated to use. Six persons found interesting and innovative. Eleven had expected to control the AR world instead of the avatar and first steered into the wrong direction. Two criticized a lack of precision, while five criticized the sensitive reactions of the avatar to even slight camera movements. 15 test persons found it appropriate for the given application and preferred it over the touchplane or joystick input. This may be based on the fact that they could use their fingers for the fight interaction as they were not needed for the motion control. Four participants suggested a stabilization of camera movements, five wished for a larger fighting area. 
Touchplane Interaction. Twelve of the participants called the touchplane easy, beginner-friendly or intuitive. Eight of the testers named a flaw of this input method: As the player taps on a point on the screen and then waits until the avatar has reached this position, the control method is not seen as suitable for games that require a high degree of response from the player. Seven mentioned the occlusion when tapping on the screen. Three said it was difficult to control the rotation of the avatar, which is important when attacking. The control method was rated as well-known from point and click games by three participants. Only six test persons gave the touchplane as their favorite input method. The analysis of the survey results and the interviews shows that an easy and intuitive control method may not necessarily be the most favoured by a player.

Joystick Interaction. Interviews show that the joystick is the most popular control of the three presented methods. 16 users described this method as easily understandable because it is simple and precise. Seven participants also mentioned the partial occlusion of the screen by the control elements as disadvantage. In addition, missing multitouch functionality of the test devices prevented simultaneous control of the character's movement and initiating an attack. 17 test persons regarded this as annoying and aggravating. Five test persons were irritated by having to use both hands for the game, one for the attack button and one for the character control. Two participants suggested that the joystick should appear at the contact position of the finger on the screen. Most of the test persons would also have appreciated a higher movement speed of the character. With the exception of one test person the mapping of the movement speed to the drag distance of the virtual joystick was considered as annoying and restrictive. Four wished for some kind of haptic feedback when the finger left the outer edge of the joystick area. The overall personal ranking of the control methods shows that the joystick is clearly preferred to the touchplane or the crosshair. 22 test subjects voted the joystick control as the most suitable input method for this kind of game.

Additional Feedback. Some of the criticism expressed by the participants was referring to the game itself and not the used control method. Missing haptic feedback was criticized as well as a lack of control over the avatar's rotation. Several users had little or no experience with AR applications and needed to get used to it first which made it difficult to differentiate between problems they had with the application and issues with the control method. Many participants asked for an input method based on physical control elements such as buttons. Unfortunately those are not available on all devices. Users preferred control methods which did not occlude the screen by the user's hands or virtual control elements. Extra features such as a dash function to speed up the characters movement or a jump function as well as additional levels were also mentioned to improve the overall game experience. 


\section{Conclusion}

We evaluated three different interaction concepts for controlling a character in an augmented reality game using a questionnaire about the intuitive use of the control methods and with personal interviews about the individual preferences of the test persons. We found a significant difference between the intuitive usability of the touchplane and the crosshair input method, but in the interviews the test persons did not prefer the touchplane method over the joystick although it had the highest QUESI score. In the interviews some players indicated that they preferred the joystick because it was well known. Also the perceiverd error rate was higher for the touchplane which might have frustrated users and let the joystick seem better suited for this kind of game. Future research needs to be conducted to implement the suggested improvents and to measure their effect on the game experience.

\section{References}

1. Naumann, A., Hurtienne, J.: Benchmarks for intuitive interaction with mobile devices. In: Proceedings of MobileHCI 2010. ACM, New York (2010)

2. Vajk, T., Coulton, P., Bamford, W., Edwards, R.: Using a mobile phone as a "wiilike" controller for playing games on a large public display. International Journal of Computer Games Technology (2007)

3. Chehimi, F., Coulton, P.: Motion controlled mobile 3d multiplayer gaming. In: Proceedings of ACE 2008. ACM, New York (2008)

4. Duh, H.B.L., Chen, V.H.H., Tan, C.B.: Playing different games on different phones: an empirical study on mobile gaming. In: Proceedings of MobileHCI 2010. ACM, New York (2008)

5. Oda, O., Lister, L.J., White, S., Feiner, S.: Developing an augmented reality racing game. In: Proceedings of INTETAIN 2008. ICST, Brussels (2007)

6. Hürst, W., van Wezel, C.: Multimodal interaction concepts for mobile augmented reality applications. In: Lee, K.-T., Tsai, W.-H., Liao, H.-Y.M., Chen, T., Hsieh, J.-W., Tseng, C.-C. (eds.) MMM 2011 Part II. LNCS, vol. 6524, pp. 157-167. Springer, Heidelberg (2011)

7. Lagerstam, E., Olsson, T., Harviainen, T.: Children and intuitiveness of interaction: a study on gesture-based interaction with augmented reality. In: Proceedings of MindTrek 2012, MindTrek 2012. ACM, New York (2012)

8. Gu, J., Duh, H.B., Kitazawa, S.: A Platform for Mobile Collaborative Augmented Reality Game: A Case Study of "AR Fighter". In: Recent Trends of Mobile Collaborative Augmented Reality Systems. Springer, New York (2011)

9. Picklum, M., Modzelewski, G., Knoop, S., Lichtenberg, T., Dittmann, P., Böhme, T., Fehn, V., John, C., Kenkel, J., Krieter, P., Niethen, P., Pampuch, N., Schnelle, M., Schwarte, Y., Stark, S., Steenbergen, A., Stehr, M., Wielenberg, H., Yildirim, M., Yüzüncü, C., Pollmann, F., Wenig, D., Malaka, R.: Player control in a realtime mobile augmented reality game. In: Herrlich, M., Malaka, R., Masuch, M. (eds.) ICEC 2012. LNCS, vol. 7522, pp. 393-396. Springer, Heidelberg (2012)

10. Weathington, B., Cunningham, C., Pittenger, D.: 14 - Correlated-Groups Designs. In: Research methods for the behavioral and social sciences. Wiley (2010) 